\title{
ON GENERALIZED NUMERICAL RANGES
}

\author{
S. M. Patel
}

The Luecke's class of operators $T$ on a Hilbert space $H$ for which $\left\|(T-v I)^{-1}\right\|=1 / d(v, W(T)), \quad v \notin \operatorname{CLW}(T), \quad$ where $\operatorname{CLW}(T)$ is the closure of the numerical range $W(T)$ of $T$, has been generalized by using the concept of generalized numerical ranges due to $\mathrm{C}$. S. Lin. Also it has been shown that the notions of generalized Minkowski distance functionals and generalized numerical ranges arise in a natural way for elements of the Calkin algebra.

Introduction. Throughout this note, by an operator, we mean a bounded linear transformation of a Hilbert space $H$ into itself. Let $B(H)$ be the Banach algebra of all operators on $H$ and $K(H)$, the closed two sided ideal of compact operators in $B(H)$. Let $\sigma(T), \operatorname{CLW}(T)$, $r(T)$ and $|W(T)|$ denote respectively the spectrum, the closure of the numerical range $W(T)$, the spectral radius and the numerical radius of an operator $T$. Con $S$ and Bdry $S$ will denote respectively the convex hull and the boundary of a subset $S$ of the complex plane $C$. We write $d(v, S)$ to denote the distance of $v$ from $S$.

Let $\hat{T}$ be the canonical image of $T$ in the (Calkin) quotient algebra $B(H) / K(H)$. For $T$ in $B(H)$, the spectrum $\sigma(\hat{T})$ and the numerical range $W_{e}(T)$ of $\hat{T}$ will be called the essential spectrum and the essential numerical range of $T$. We write $r_{e}(T)$ to denote the spectral radius of $\hat{T}$. Salinas [7, Lemma 2.2] has shown that $r_{e}(T)=\inf \{r(T+K)$ : $K \in K(H)\}$.

Let $C_{\rho}$ be the class of operators with unitary $\rho$-dilation in the sense of B. Sz-Nagy and C. Foias [5]. In [1], Holbrook has defined generalized Minkowski distance functionals $w_{\rho}(\cdot)(0 \leqq \rho<\infty)$ on $B(H)$ as $w_{\rho}(T)=\inf \left\{u: u>0\right.$ and $\left.u^{-1} T \in C_{\rho}\right\}$.

We list in the following theorem some of the properties of $w_{\rho}(\cdot)$ which we shall need in the sequel:

THEOREM A (Holbrook [1]). $w_{\rho}(\cdot)$ has the following properties:

(1) $w_{\rho}(T)<\infty$;

(2) $w_{\rho}(T)>0$ unless $T=0$, in fact, $w_{\rho}(T) \geqq 1 / \rho\|T\|$;

(3) $w_{\rho}(v T)=|v| w_{\rho}(T)$ for $v \in C$;

(4) The function $w_{\rho}(\cdot)$ is a norm on $B(H)$ whenever $0<\rho \leqq 2$;

(5) For each $\rho>0$ and $T \in B(H), \quad w_{\rho}\left(T^{k}\right) \leqq w_{\rho}(T)^{k}, \quad k=$ $1,2,3, \cdots ;$

(6) $w_{\rho}(T)$ is a continuous and nonincreasing function of $\rho$; 
(7) $\quad r(T)=\lim _{\rho \rightarrow \infty} w_{\rho}(T)$;

(8) $K_{\rho} r(T) \leqq w_{\rho}(T) \leqq K_{\rho}\|T\|$, where $K_{\rho}=1$ or $2 / \rho-1$ according as $\rho \geqq 1$ or $\rho<1$.

Using the concept of $w_{\rho}(\cdot)$, C. S. Lin [3] has recently defined a new concept of generalized numerical ranges $W_{\rho}(T)$ of $T$ as

$$
W_{\rho}(T)=\cap\left\{u:|u-v| \leqq w_{\rho}(T-v I), u, v \in C\right\}, \quad 1 \leqq \rho<\infty .
$$

For a ready reference, we state some of the results about $W_{\rho}(T)$ from [3].

THEOREM B [3]. $W_{\rho}(T)$ has the following properties:

1. $W_{\rho}(T)$ is a compact convex subset of $C$;

2. Con $\sigma(T) \subseteq W_{\rho}(T)$ and $W_{x}(T)=\operatorname{Con} \sigma(T)$;

3. $W_{\rho}(c T+b I)=c W_{\rho}(T)+b, b, c \in C$;

4. $W_{\rho}(T) \supseteq W_{\rho^{\prime}}(T)$ for $\rho^{\prime}>\rho$; in particular, $W_{\rho}(T) \subseteq \operatorname{CLW}(T)$ for $\rho \geqq 1 ;$

5. $W_{\rho}(T)=\operatorname{CLW}(T)$ for $1 \leqq \rho \leqq 2$.

The object of this article is to pursue further the study of generalized numerical ranges. In $\S 1$, we investigate the properties of those operators $T$ for which $w_{\alpha}\left((T-v I)^{-1}\right) \geqq 1 / d\left(v, W_{\rho}(T)\right)$ for all $v \notin W_{\rho}(T)$, where $\alpha \geqq 1$. Also we introduce in $\$ 2$, the notions of generalized Minkowski distance functionals and generalized essential numerical ranges for elements of the Calkin algebra.

1. In [4], Luecke defines a subclass $\mathscr{R}$ of convexoid operators as follows:

$\mathscr{R}=\left\{T \in B(H):\left\|(T-v I)^{-1}\right\|=1 / d(v, W(T))\right.$ for all $\left.v \notin \operatorname{CLW}(T)\right\}$

Our first observation for such operators $T$ is the following

THEOREM 1. TER if and only if

$$
w_{\alpha}\left((T-v I)^{-1}\right)=1 / d\left(v, W_{\rho}(T)\right)
$$

for all $v \notin W_{\rho}(T)$, where $\alpha \geqq 1, \rho \geqq 1$.

Proof. Since $T \in \mathscr{R}$ if and only if Bdry $W(T) \subseteq \sigma(T)$ [4], it follows that for such operators $1 / d(v, W(T))=1 / d(v, \sigma(T)) \leqq w_{\alpha}\left((T-v I)^{-1}\right) \leqq$ $\left\|(T-v I)^{-1}\right\| \leqq 1 / d(v, W(T))$ or $w_{\alpha}\left((T-v I)^{-1}\right)=1 / d(v, W(T))$ for all $v \notin \mathrm{CLW}(T)$, where $\alpha \geqq 1$. Now the convexoidity of $T$ implies $\operatorname{CLW}(T)=\operatorname{Con} \sigma(T) \subseteq W_{\rho}(T)$, and hence by Theorem $\mathrm{B}(4), \operatorname{CLW}(T)=$ 
$W_{\rho}(T)$ for all $\rho \geqq 1$. Thus $w_{\alpha}\left((T-v I)^{-1}\right)=1 / d\left(v, W_{\rho}(T)\right)$ for all $v \notin W_{\rho}(T)$, where $\alpha \geqq 1, \rho \geqq 1$.

Author [6] has shown that a closed convex subset $M$ of $C$ contains $\operatorname{CLW}(T)$ if and only if $w_{\alpha}\left((T-v I)^{-1}\right) \leqq 1 / d(v, M)$ for all $v \notin M$, where $\alpha \geqq 1$. Therefore if $T$ satisfies $(*)$, then $\operatorname{CLW}(T) \subseteq W_{\rho}(T)$. But by Theorem $\mathrm{B}(4), W_{\rho}(T) \subseteq \mathrm{CLW}(T)$, and so $\operatorname{CLW}(T)=W_{\rho}(T)$. Thus $(*)$ reduces to $w_{\alpha}\left((T-v I)^{-1}\right)=1 / d(v, W(T))$ and hence $\left\|(T-v I)^{-1}\right\|=$ $1 / d(v, W(T))$ for all $v \notin \mathrm{CLW}(T)$. This shows that $T \in \mathscr{R}$.

Taking a clue from these conclusions, we generalize operators of class $\mathscr{R}$ as follows

DEFInITION. Let $\alpha \geqq 1, \rho \geqq 1$. An operator $T$ is said to be an operator of class $\mathscr{R}_{\rho}$ if for all $v \notin W_{\rho}(T), w_{\alpha}\left((T-v I)^{-1}\right) \geqq 1 / d\left(v, W_{\rho}(T)\right)$.

In view of Theorem $\mathrm{B}(4),(5)$, it is not difficult to see that $\mathscr{R}_{\rho} \subseteq \mathscr{R}_{\rho^{\prime}}$ whenever $\rho<\rho^{\prime}$; in particular, $\mathscr{R} \subseteq \mathscr{R}_{\rho}$ for any $\rho \geqq 1$. Moreover for $1 \leqq \rho \leqq 2, \mathscr{R}=\mathscr{R}_{\rho}$.

We now characterize operators of class $\mathscr{R}_{\rho}$ in the following theorem which also shows that our definition of $\mathscr{R}_{\rho}$ is, in fact, independent of $\alpha$.

\section{THEOREM 2. $T \in \mathscr{R}_{\rho}$ if and only if $\operatorname{Bdry} W_{\rho}(T) \subseteq \sigma(T)$.}

Proof. Suppose first that Bdry $W_{\rho}(T) \subseteq \sigma(T)$. Then for $v \notin W_{\rho}(T), \quad d\left(v, W_{\rho}(T)\right)=d(v, \sigma(T))$ and hence $1 / d\left(v, W_{\rho}(T)\right)=$ $1 / d(v, \sigma(T)) \leqq w_{\alpha}\left((T-v I)^{-1}\right)$ or $T \in \mathscr{R}_{\rho}$. On the other hand, if $v \in$ Bdry $W_{\rho}(T)$, then we can find a sequence $\left\{v_{n}\right\}$ of complex numbers such that $\quad v_{n} \rightarrow v$ and $\left|v_{n}-v\right|=d\left(v_{n}, W_{\rho}(T)\right)>0$ for $n=1,2,3, \cdots$. Therefore if $T \in \mathscr{R}_{\rho}$ then $w_{\alpha}\left(\left(T-v_{n} I\right)^{-1}\right) \rightarrow \infty$ and hence $v \in \sigma(T)$; thus Bdry $W_{\rho}(T) \subseteq \sigma(T)$.

Following Lin [3], we call $T$ to be a $\rho$-convexoid operator if $W_{\rho}(T)=\operatorname{Con} \sigma(T)$. Let $\mathscr{L}_{\rho}$ be the class of such operators. Then $\mathscr{L}_{\rho} \subseteq \mathscr{L}_{\rho^{\prime}}$ for $\rho^{\prime}>\rho$ and $\mathscr{L}_{\rho}$ consists of all convexoid operators whenever $1 \leqq \rho \leqq 2$; in particular the class of convexoid operators is contained in $\mathscr{L}_{\rho}$ for all $\rho \geqq 1$.

\section{Corollary 1. $\mathscr{R}_{\rho} \subseteq \mathscr{L}_{\rho}$ and $\mathscr{R}_{\rho} \neq \mathscr{L}_{\rho}$.}

Proof. If $T \in \mathscr{R}_{\rho}$, then by Theorem $2, W_{\rho}(T)=\operatorname{con} \sigma(T)$. This proves the first assertion. To prove the remaining assertion, it suffices to take a convexoid operator $T$ whose spectrum is finite but contains more than one point. Then $T \in \mathscr{L}_{\rho}$ for all $\rho \geqq 1$. However, in view of Theorem 2, $T$ cannot be in any $\mathscr{R}_{\rho}$.

As observed earlier, the class $\mathscr{R}$ is contained in the class $\mathscr{R}_{\rho}$ for $\rho \geqq 1$. Our next result shows that the class $\mathscr{R}_{\rho}$ is indeed larger than the class $\mathscr{R}$ for any $\rho>2$. 
THEOREM 3. There exists a nonconvexoid operator which is of class $\mathscr{R}_{\rho}$ for any $\rho>2$.

Proof. Let $A=\left[\begin{array}{ll}0 & 1 \\ 0 & 0\end{array}\right]$. We claim that $W_{\rho}(A) \neq \operatorname{CLW}(A)$, for $\rho>2$. If not, then $w_{\rho} \circ(A)=|W(A)|$ where $w_{\rho}^{\circ}(A)=\sup \{|\lambda|$ : $\left.\lambda \in W_{\rho}(A)\right\}$. Since $w_{\rho}(A) \geqq w_{\rho} \circ(A)$ and $|W(A)| \geqq w_{\rho}(A)$, we have $w_{\rho}(A)=|W(A)|$, which is not possible as $w_{\rho}(A)=1 / \rho$ and $|W(A)|=$ $1 / 2[1]$. Thus $W_{\rho}(A) \neq \operatorname{CLW}(A)$ for any $\rho>2$.

Let $N$ be a normal operator such that $\sigma(N)=W_{\rho}(A)$. Clearly

$$
r(N-v I) \leqq w_{\rho}(A-v I) \text { for all } v \in C
$$

Let $T=N \oplus A$, then $\operatorname{CLW}(T)=\operatorname{Con}\{\operatorname{CLW}(N) \cup \operatorname{CLW}(A)\}=$ $\operatorname{Con}\{\operatorname{Con} \sigma(N) \cup \operatorname{CLW}(A)\}=\operatorname{Con}\left\{W_{p}(A) \cup \operatorname{CLW}(A)\right\}=\operatorname{CLW}(A)$.

Thus

$$
C L W(T)=\operatorname{CLW}(A) .
$$

Also $\sigma(T)=\sigma(N) \cup \sigma(A)=W_{\rho}(A) \cup \sigma(A)=W_{\rho}(A)$. Hence

$$
\operatorname{Con} \sigma(T)=W_{\rho}(A)=\sigma(T) \text {. }
$$

Since $W_{\rho}(A) \neq \operatorname{CLW}(A)$, it follows from (2) and (3) that CLW $(T)$ $\neq \operatorname{Con} \sigma(T)$, which shows that $T$ is nonconvexoid. On the other hand

$$
\begin{aligned}
W_{\rho}(T) & =\bigcap_{v}\left\{u:|u-v| \leqq w_{\rho}(T-v I)\right\} \\
& =\bigcap_{v}\left\{u:|u-v| \leqq \max \left\{w_{\rho}(N-v I), w_{\rho}(A-v I)\right\}\right\}
\end{aligned}
$$

$[2$, Theorem 4.1]

$$
\begin{aligned}
& =\bigcap_{v}\left\{u:|u-v| \leqq \max \left\{r(N-v I), w_{\rho}(A-v I)\right\}\right\} \\
& =\bigcap_{v}\left\{u:|u-v| \leqq w_{\rho}(A-v I)\right\} \quad \text { (by (1)). }
\end{aligned}
$$

So $W_{\rho}(T)=W_{\rho}(A)$. This together with (3) implies that $T \in \mathscr{R}_{\rho}$. This completes the proof.

It has been established in [4] that the inverse of a nonsingular operator of class $\mathscr{R}$ is not necessarily of class $\mathscr{R}$. To show that the corresponding assertion for operators of class $\mathscr{R}_{\rho}$ is also true, we prove 
THEOREM 4. There exists a nonsingular operator $T$ in $\cap \mathscr{R}_{\rho}$ such that $T^{-1} \notin \cup \mathscr{R}_{\rho}$.

Proof. Let $W$ be the simple bilateral shift on $H$. Then $T=1 / 2$ $\bigoplus W$ will be an operator on $H \oplus H$ such that $\sigma(T)=\{1 / 2\}$ $\cup\{v:|v|=1\}$, and $\operatorname{CLW}(T)=\{v:|v| \leqq 1\}$. Since Bdry $W(T) \subseteq \sigma(T)$, $T \in \mathscr{R} \subseteq \mathscr{R}_{\rho} . \quad$ Now $\quad \sigma\left(T^{-1}\right)=\{2\} \cup\{v:|v|=1\} . \quad$ Clearly $1+1 / 2 i \in$ Bdry Con $\sigma\left(T^{-1}\right)$. If $T^{-1} \in \mathscr{R}_{\rho}$ then $\operatorname{Bdry} W_{\rho}\left(T^{-1}\right)=\operatorname{Bdry} \operatorname{Con} \sigma\left(T^{-1}\right)$ $\subseteq \sigma\left(T^{-1}\right)$. Consequently, $1+1 / 2 i \in \sigma\left(T^{-1}\right)$, which is not true. Thus $T^{-1} \notin \cup \mathscr{R}_{\rho}$.

2. In the Calkin algebra, the norm of $\hat{T}$ is defined by

$$
\|\hat{T}\|=\inf \{\|T+K\|: K \in K(H)\} .
$$

This provides us with a clue to introduce the concept of generalized Minkowski distance functionals $w_{\rho}(\cdot)$ on this algebra in the following manner:

$$
w_{\rho}(\hat{T})=\inf \left\{w_{\rho}(T+K) ; K \in K(H)\right\}
$$

Some of the properties of these functionals are just on the surface and follow from those of $w_{\rho}(T)$ listed in Theorem A. erties:

TheOREM 5. $w_{\rho}(\cdot)$ defined on $B(H) / K(H)$ has the following prop-

(1) $w_{\rho}(\hat{T})<\infty$;

(2) $1 / \rho\|\hat{T}\| \leqq w_{\rho}(\hat{T})$;

(3) $K_{\rho} r_{e}(T) \leqq w_{\rho}(\hat{T}) \leqq K_{\rho}\|\hat{T}\|$, where $K_{\rho}=1$ or $2 / \rho-1$ according as $\rho \geqq 1$ or $\rho<1$;

(4) $w_{\rho}(v \hat{T})=|v| w_{\rho}(\hat{T})$ for $v \in C$;

(5) $w_{\rho}(\cdot)$ is a norm on $B(H) / K(H)$, whenever $0<\rho \leqq 2$;

(6) $w_{\rho}(T)$ is continuous and nonincreasing function of $\rho$;

(7) $\lim _{\rho \rightarrow \infty} w_{\rho}(\hat{T})=r_{e}(T)$;

(8) $w_{\rho}\left(\hat{T}^{n}\right) \leqq w_{\rho}(\hat{T})^{n}$ for $n=1,2,3 \cdots$.

Next we define the generalized essential numerical ranges $W_{\rho}(\hat{T})$ ( $\rho \geqq 1$ ) of $T$ as follows:

$$
W_{\rho}(\hat{T})=\cap\left\{u:|u-v| \leqq w_{\rho}(\hat{T}-v \hat{I}), u, v \in C\right\}
$$

It is easy to show that $W_{\rho}(\hat{T})$ is a compact convex subset of $C$. Since $w_{\rho}(\hat{T}) \leqq w_{\rho}(T), \quad W_{\rho}(\hat{T}) \subseteq W_{\rho}(T)$. Also by Theorem 4(6), $W_{\rho}(\hat{T}) \supseteq$ $W_{\rho^{\prime}}(\hat{T})$ whenever $\rho^{\prime}>\rho$. Following the same argument that was used in 
the proof of Theorem $\mathrm{B}(3)$, one can show that $W_{\rho}(c \hat{T}+b \hat{I})=c W_{\rho}(\hat{T})+b$, $b, c \in C$. The relation $\operatorname{Con} \sigma(\hat{T})=\cap\{u:|u-v| \leqq$ $\left.r_{e}(T-v I), u, v \in C\right\}$, along with Theorem 4(3), (7) gives Con $\sigma(\hat{T}) \subseteq$ $W_{\rho}(\hat{T})$ and $W_{\infty}(\hat{T})=$ Con $\sigma(\hat{T})$. It is immediate from the definition that $u \in W_{\rho}(\hat{T})$ if and only if $|u-v| \leqq w_{\rho}(T+K-v I)$ for all $v$ in $C$ and for $K$ in $K(H)$. Thus $W_{\rho}(\hat{T})=\cap W_{\rho}(T+K)$, where the intersection is taken over all $K$ in $K(H)$. In particular, $W_{\rho}(\hat{T})=W_{e}(T)$ for $1 \leqq \rho \leqq 2$ in view of Theorem $\mathrm{B}(5)$ and [8, Theorem 9]. We summarize all these conclusions in

THEOREM 6. $W_{\rho}(\hat{T})$ has the following properties:

(1) $W_{\rho}(\hat{T})$ is a compact convex subset of $C$;

(2) $W_{\rho}(\hat{T}) \subseteq W_{\rho}(T)$ and for $\rho^{\prime}>\rho, W_{\rho^{\prime}}(\hat{T}) \subseteq W_{\rho}(\hat{T})$;

(3) $W_{\rho}(c \hat{T}+b \hat{I})=c W_{\rho}(\hat{T})+b, b, c \in C$;

(4) $W_{x}(\hat{T})=\operatorname{Con} \sigma(\hat{T}) \subseteq W_{\rho}(\hat{T})$;

(5) $W_{\rho}(\hat{T})=\cap W_{\rho}(T+K)$; in particular $W_{\rho}(\hat{T})=W_{e}(T)$ for $1 \leqq$ $\rho \leqq 2$, and $W_{\rho}(\hat{T})=W_{e}(T)$ for all $\rho \geqq 1$.

REMARK. In the light of the above theorem, it is natural to ask the following question: Is it true that $W_{\rho^{\prime}}(\hat{T})$ is properly contained in $W_{\rho}(\hat{T})$ for $\rho^{\prime}>\rho$ ? If $\hat{T}$ is convexoid (i.e. $W_{e}(\hat{T})=$ Con $\sigma(\hat{T})$ ), the answer is in negative. This will follow easily from Theorem 5(4), (5). The question still remains open in case $\hat{T}$ is nonconvexoid. However, in particular, if $\rho=2$ then for a nonconvexoid $\hat{T}$, there does exist $\rho^{\prime}>2$ such that $W_{\rho^{\prime}}(\hat{T})$ is properly contained in $W_{\rho}(\hat{T})=W_{e}(T)$; for if not, then $W_{\rho^{\prime}}(\hat{T})=W_{e}(T)$ for all $\rho>2$ together with Theorem 5(4) would imply that $\operatorname{Con} \sigma(\hat{T})=$ $W_{\infty}(\hat{T})=W_{e}(T)$ and hence that $\hat{T}$ is convexoid.

Acknowledgement. The author is indebted to Dr. B. S. Yadav for his help in the preparation of this article. Also he is thankful to the referee for his suggestions to improve the original version of the present paper.

\section{REFERENCES}

1. J. A. R. Holbrook, On the power bounded operators of Sz-Nagy and Foias, Acta Sci. Math., 29 (1968), 299-310.

2. - Inequalities governing the operator radii associated with unitary $\rho$-dilations, Michigan Math. J., 18 (1971), 149-159.

3. C. S. Lin, On a family of generalized numerical ranges, Canad. J. Math., XXVI, No. 3 (1974), $678-685$.

4. G. R. Luecke, A class of operators on Hilbert space, Pacific J. Math., 41 (1971), 153-156.

5. B. Sz-Nagy and C. Foias, Harmonic Analysis of Operators on Hilbert Space, North Holland Publishing Co., (Amsterdam-London, 1970). 
6. S. M. Patel, On some classes of operators associated with operator radii of Holbrook, 39th Conference of Indian Math. Soc. (1973).

7. N. Salinas, Operators with essentially disconnected spectrum, Acta Sci. Math., 33 (1972), 193-205.

8. J. G. Stampfli and J. P. Williams, Growth conditions and the numerical range in a Banach algebra, Tohoku Math. J., 20 (1968), 417-424.

Received February 6, 1975, and in revised form April 2, 1976.

UNIVERSITY OF DELHI

Current Address: Department of Mathematics,

SARDAR PATEl UNIVERSITY,

VAllabH Vidyanagar-388 120.

GuJARAT (INDIA) 
